\title{
Noninfectious lung complications after allogeneic haematopoietic stem cell transplantation
}

\author{
Anne Bergeron ${ }^{1,2}$, Sylvie Chevret ${ }^{2,3}$, Régis Peffault de Latour ${ }^{4}$, \\ Karine Chagnon ${ }^{1,5}$, Constance de Margerie-Mellon $\mathbb{1}^{6}$, Frédéric Rivière ${ }^{1}$, \\ Marie Robin ${ }^{4}$, Jean Mani ${ }^{7}$, Gwenael Lorillon ${ }^{1}$, Gérard Socié ${ }^{4,8}$ and \\ Abdellatif Tazi ${ }^{1,2,8}$
}

Affiliations: ${ }^{1}$ AP-HP, Hôpital Saint-Louis, Service de Pneumologie, Paris, France. ${ }^{2}$ Université Paris Diderot, Sorbonne Paris Cité, UMR 1153 CRESS, Biostatistics and Clinical Epidemiology Research Team, Paris, France. ${ }^{3}$ AP-HP, Hôpital Saint-Louis, Service de Biostatistique et Information Médicale, Paris, France. ${ }^{4}$ Université Paris Diderot, Sorbonne Paris Cité, AP-HP, Hématologie-Greffe, Hôpital Saint-Louis, Paris, France. ${ }^{5}$ Université de Montréal, Service de Pneumologie, Hôpital Maisonneuve-Rosemont, Montréal, QC, Canada. ${ }^{6}$ Université Paris Diderot, Sorbonne Paris Cité, AP-HP, Radiologie, Hôpital Saint-Louis, Paris, France. ${ }^{7}$ Département d'Imagerie Clinique du Sport, Paris, France. ${ }^{8}$ These two authors contributed equally to this work.

Correspondence: Anne Bergeron, Service de Pneumologie, Hôpital Saint-Louis, 1 avenue Claude Vellefaux, 75475 Paris Cedex 10, France. E-mail: anne.bergeron-lafauriedaphp.fr

@ERSpublications

Chest irradiation, pneumonia and low FEF25-75\% predict lung complications after allogeneic bone marrow transplantation http://ow.ly/pGel30j61Da

Cite this article as: Bergeron A, Chevret S, Peffault de Latour R, et al. Noninfectious lung complications after allogeneic haematopoietic stem cell transplantation. Eur Respir J 2018; 51: 1702617 [https://doi.org/ 10.1183/13993003.02617-2017].

ABSTRACT Epidemiological data on late-onset noninfectious pulmonary complications (LONIPCs) following allogeneic haematopoietic stem cell transplantation (HSCT) are derived exclusively from retrospective studies and are conflicting. We aimed to evaluate prospectively the incidence, risk factors and outcomes for LONIPCs.

All consecutive patients scheduled to receive allogeneic HSCT between 2006 and 2008 at a university teaching hospital in France were screened for inclusion in the study. Eligible patients were those surviving at day 100. Among 243 screened patients, 198 patients were included in the analysis. The median (interquartile range) follow-up was 72.3 (15.2-88.5) months. 55 LONIPCs were diagnosed in 43 patients. Bronchiolitis obliterans syndrome $(n=22)$ and interstitial lung disease $(n=12)$ were the most common LONIPCs. At 36 months after inclusion, the estimated cumulative incidence of LONIPCs was 19.8\% (95\% CI 14.2-25.3\%). The estimated median survival after the diagnosis of LONIPCs was 78.5 months (95\% CI 20.0-not reached). Based on a multivariate Cox model, a history of chest irradiation anytime prior to HSCT, a history of pneumonia within 100 days post-HSCT and a low mean forced expiratory flow at 25$75 \%$ of forced vital capacity at day 100 were associated with the development of LONIPCs.

Our data provide clues to identify patients at high risk of developing LONIPCs. These patients should be targeted for close monitoring to provide earlier LONIPC treatment or prophylactic treatment.

This article has supplementary material available from erj.ersjournals.com

This study is registered at ClinicalTrials.gov with identifier number NCT01219972.

Received: April 192017 | Accepted after revision: March 142018

Copyright $\odot$ ERS 2018 


\section{Introduction}

Late-onset noninfectious pulmonary complications (LONIPCs) that occur beyond the third month following allogeneic haematopoietic stem cell transplantation (HSCT) have a significant effect on patient outcomes, with high associated mortality and morbidity rates [1-3]. Most of these lung complications that have been associated with chronic graft versus host disease (GVHD) mainly occur during the first year after HSCT and rarely occur beyond 2 years [3-6]. Although lung biopsy is the gold standard to classify most LONIPCs, fewer patients undergo lung biopsy, and the diagnosis mostly now relies on pulmonary function testing (PFT) and lung computed tomography (CT) scanning [7].

Findings from the available studies regarding both the incidence and risk factors for LONIPCs show many discrepancies. The reported incidence of LONIPCs ranges from $10 \%$ to $26 \%[1,2,7-11]$, while contradictory findings have been reported regarding the predictive role of the conditioning regimen, GVHD or stem cell sources [1-3, 7-11]. The main reason for these discrepancies is that all previous studies were retrospective, leading to significant biases, including incomplete data, an absence of detailed lung function and a lack of clinical correlation with most of the studies focused on PFT. In addition, most studies reviewed long periods characterised by variations in PFT screening strategies and diagnostic criteria for LONIPCs and HSCT procedures. Furthermore, although recommended, adherence to PFT guidelines in HSCT recipients is poor in routine practice and leads to missing data [12].

Specific limitations can be addressed for studies focused on bronchiolitis obliterans syndrome (BOS), which is the most frequent LONIPC. One of the main limitations is based on the difficulty in determining an accurate PFT definition for BOS to compare studies. Indeed, prior to the 2005 National Institutes of Health (NIH) consensus diagnostic criteria for BOS [13], various PFT criteria were used. Even after 2005, some authors used modified criteria to improve their sensitivity $[4,14,15]$. Recent studies have also shown that regardless of the diagnostic criteria of BOS, a significant proportion of patients experience a significant decline in forced expiratory volume in $1 \mathrm{~s}$ (FEV1) after HSCT [12]. Data suggest that this decline could be a good predictor for the development of subsequent BOS.

In addition to PFT, lung CT is an essential diagnostic tool for pulmonary complications. The evidence of air trapping by expiratory CT scan, small airway thickening or bronchiectasis are part of the BOS diagnostic criteria [15]. Whether any CT scan sign can predict the occurrence of post-HSCT BOS or other LONIPCs remains unknown.

Improved specification by LONIPCs and identification of early risk factors in a prospective study are mandatory to focus on high-risk allogeneic HSCT recipients to achieve an earlier diagnosis, initiate earlier treatment and evaluate prophylactic strategies to improve prognoses.

\section{Methods}

\section{Study design and participants}

In this observational prospective cohort study, all consecutive patients who were scheduled to receive an allogeneic HSCT between January 31, 2006 and December 31, 2008 at the Hôpital Saint-Louis (Paris, France) university teaching hospital were screened for inclusion within the 2 weeks before transplantation. Those allogeneic HSCT recipients surviving at day 100 in the absence of early relapse of haematological disease or refusal were included in the cohort. This study was approved by a central institutional review board (Comité Consultatif de Protection des Personnes dans la Recherche Biomédicale Paris Saint-Louis). All of the patients or legal guardians of the patients $<18$ years of age provided written informed consent before transplant, although definite inclusion occurred at day 100 after checking all inclusion and exclusion criteria. The trial was registered at ClinicalTrials.gov (identifier NCT01219972).

\section{Procedures}

At inclusion (day 100), the history of the underlying haematological disease, the characteristics of the transplant and the occurrence of GVHD in the first 100 days after HSCT were recorded.

PFT, lung high-resolution CT (HRCT) scans and a clinical assessment by a pulmonologist were performed sequentially before transplant, at inclusion, and then at $6,12,18,24$ and 36 months after HSCT. Thereafter, PFT was performed annually. Additional explorations were performed in the case of new-onset respiratory symptoms. PFT was performed using a body plethysmograph (Jaeger Masterscreen Body; CareFusion Germany 234, Hoechberg, Germany). Predictive values were determined as previously described [16]. As a pre-transplant lung function score (LFS) was previously associated with allogeneic HSCT recipient outcomes, LFS was determined [17].

HRCT scans were performed on every patient using a multislice CT scanner (Optima 660CT with low dose device; GE Healthcare, Milwaukee, WI, USA). Helicoidal acquisitions were acquired during both deep inspiration and expiration. The images were reconstructed using a high-spatial-frequency algorithm. One 
radiologist (C.d.M-M.) and two experienced pulmonary physicians (A.B. and K.C.) who were unaware of both the clinical and PFT results reviewed the CT scans and reached a conclusion by consensus. On the expiratory scans, the extent of air trapping was scored at three levels on axial images (upper, middle and lower) for each lung as: 1 (0-25\% affected), 2 (25-50\% affected), 3 (50-75\% affected) or 4 (75-100\% affected). For each lung, the score ranged from 6 to $24[18,19]$. An air trapping score $>6$ was considered clinically significant.

\section{End-points and definitions}

Although previous studies suggested that LONIPCs mainly occur during the first year after HSCT and rarely occur beyond 2 years, in our clinical experience LONIPCs could occur beyond 2 years, particularly interstitial pneumonias. Therefore, in this prospective study, we decided to choose a follow-up of 36 months to obtain a further insight into such late occurrences by providing an estimate of occurrence over time within the first 3 years of HSCT. The main end-point was thus the occurrence of LONIPCs during the first 36 months following study inclusion. LONIPCs were classified as BOS, interstitial lung disease (ILD) and "others", which included venous thromboembolic disease (VTED), air leak syndrome (i.e. pneumothorax or pneumomediastinum), exudative pleural effusion and restrictive lung defects (RLDs). Any LONIPC was considered during all of the follow-up, i.e. both at the time of planned CT scan or if diagnosed between two study visits. BOS was previously defined as: 1) an absence of respiratory infection at the time of PFT; 2) either an $\mathrm{FEV}_{1}<75 \%$ of predicted or a decline $>10 \%$ of FEV 1 from the pre-transplant value; and 3) either FEV1/vital capacity (VC) $<0.7$ or a concomitant decrease in both FEV 1 and forced VC (FVC) $<80 \%$ of predicted, with a total lung capacity (TLC) $>80 \%$ of predicted $[4,20]$. Concordance with the NIH definition was investigated for each case [13, 15]. These abnormalities persisted for two subsequent PFT measurements at 4 -week intervals. A RLD was defined as a TLC $<80 \%$ of predicted. ILD was diagnosed when diffuse opacities were present on HRCT and an infectious cause for these opacities was ruled out, as we reported previously [5]. VTED included pulmonary embolism diagnosed by CT pulmonary angiography or deep venous thrombosis diagnosed by ultrasound. Lower respiratory tract infection (LRTI) was defined as an association of fever, respiratory symptoms and a new lung infiltrate on chest radiography or lung CT scan. All these events were investigated with bronchoalveolar lavage, as described previously [5]. Proven LRTI was defined when a pathogen was documented. LRTI was defined as probable if clinical and radiological abnormalities improved with antimicrobial treatment but no pathogens were identified. Diagnostic criteria and severity of acute and extrathoracic chronic GVHD were assessed according to the NIH criteria, with a grading scale from 1 to 4 for acute GVHD [21], and the global score of mild, moderate and severe reflects the degree of organ impact and functional impairment due to chronic GVHD [15].

Secondary end-points were survival after LONIPCs and long-term time course of PFT (FEV1, FVC, TLC, mean forced expiratory flow (FEF) at 25-75\% of FVC (FEF25-75\%) and instantaneous $\mathrm{FEF}$ at $50 \%$ of $\mathrm{FVC}$ (FEF50\%)). For descriptive purposes, overall survival was also computed.

\section{Statistical analysis}

Summary statistics were reported, i.e. median (interquartile range (IQR)), unless otherwise stated. All analyses of survival data were performed using data obtained up to June 2016.

Survival after inclusion (day 100 after HSCT) and survival after LONIPCs were both estimated using the Kaplan-Meier method; a Cox model with a time-dependent variable was used to assess the impact of LONIPCs on survival. Competing-risk end-points (LONIPC, BOS, chronic GVHD and relapse) were computed from study inclusion in which patients who died free from the event of interest were considered competing-risk events. Early risk factors were defined as those present before transplantation or at inclusion (day 100). Predictive analyses of the hazard of LONIPC or BOS occurrence within the first 36 months were based on cause-specific Cox models. First, univariate models were fitted whereby all the covariates reported previously as risk factors for LONIPCs were considered fixed covariates; chronic GVHD after inclusion was tested as a time-dependent covariate. Then, multivariate models were fitted on those predictors selected from univariate analyses at the $10 \%$ level, segregating pre-transplant, transplant and post-transplant (at day 100) characteristics. Finally, multivariate models were fitted based on all potential predictors selected whatever the time of measurement at the $10 \%$ level, with model selection based on a stepwise procedure using variables associated with the outcome at the $5 \%$ level and after multiple imputations with chained equations (MICE) of missing data, averaging the estimates over 50 data sets. Sensitivity analyses based on a complete case analysis were also performed.

To describe the course of PFT over time, we used a nonparametric $k$-means algorithm for clustering longitudinal data, providing several techniques for dealing with missing values in trajectories; the number of trajectories was defined as those maximising the Calinski-Harabatz criterion as given by the $\mathrm{kml}$ 
package [22]. All estimates of cumulative incidence, survival rates and cause-specific hazard ratios were reported with $95 \%$ confidence intervals.

Statistical analyses were performed using SAS version 9.3 (SAS Institute, Cary, NC, USA) and R version 3.2.2 (www.R-project.org). Two-sided p-values $\leqslant 0.05$ indicated statistical significance.

\section{Results}

From May 2006 to April 2009, 198 out of 243 screened patients were included in the analysis (figure 1). Two (1\%) patients had FEV1/VC $<0.7$ and nine (5\%) patients had a RLD. LFS was impaired in 118 (75\%) patients. $35(23 \%)$ patients had a significant air trapping score $(>6)$ on chest CT scan. Pre-transplantation and inclusion data (day 100) are summarised in table 1.

Within 100 days following HSCT, 33 (17\%) patients developed grade $\geqslant 3$ acute GVHD (skin, n=31; liver, $\mathrm{n}=7$; gut, $\mathrm{n}=19)$ and $11(6 \%)$ patients had extensive chronic GVHD. Nine of the 18 LRTIs were documented (bacteria, $n=3$; fungus, $n=2$; virus, $n=4$ ). There were no notable differences between the patients included and not included in the study (supplementary table S1).

The median (IQR) duration of follow-up was 72.3 (15.2-88.5) months. Three patients were lost to follow-up at 5, 14 and 28 months, respectively; 40 patients relapsed; and 81 patients died (48 free of relapse and 33 after relapse), 68 within 36 months, with a 3-year survival of $65.4 \%$ (95\% CI 59.1-72.4\%) (figure 2a). In addition to relapse, patients died mostly from pulmonary causes $(n=18 ; 14$ due to infectious pneumonia and four from respiratory failure), 16 from GVHD, seven from other causes, while 11 causes of death were unknown.

55 episodes of LONIPCs were diagnosed in 43 patients (figure 1). Diagnoses included BOS ( $\mathrm{n}=22$ in 22 patients), ILD ( $\mathrm{n}=12$ in 12 patients) and others ( $\mathrm{n}=21$ in 19 patients). LONIPCs other than BOS and ILD

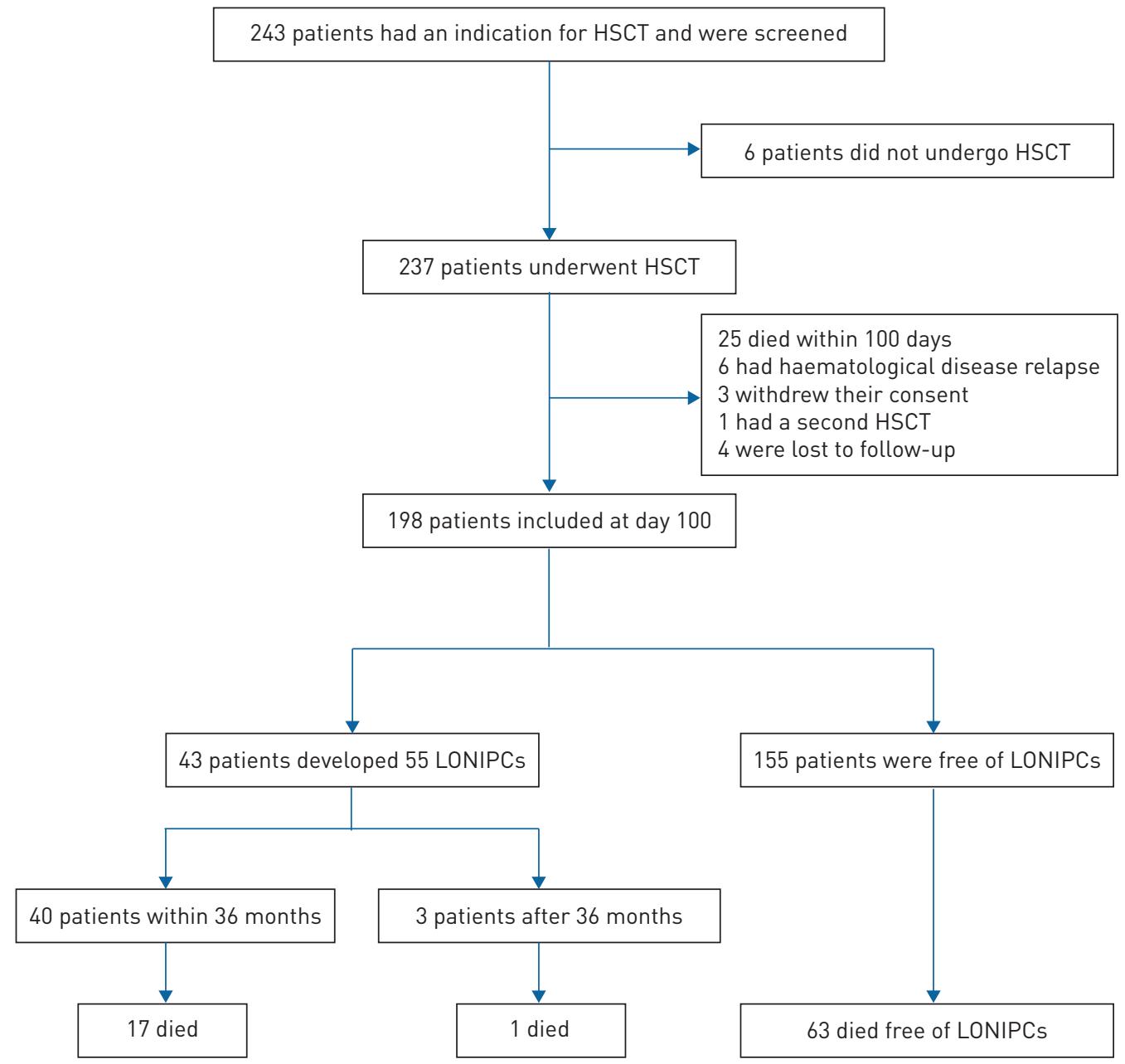

FIGURE 1 Study flowchart. HSCT: haematopoietic stem cell transplantation; LONIPC: late-onset noninfectious pulmonary complication. 
TABLE 1 Patient characteristics measured before or at the time of allogeneic haematopoietic stem cell transplantation (HSCT) and at the time of inclusion (day 100 after allogeneic HSCT)

\section{Patients}

Baseline characteristics (before transplant)

Male

$120(61)$

Age at transplant years

History of smoking

65 (33)

Underlying disease

Acute leukaemia

Myelodysplastic syndrome

Myeloproliferative syndrome

$19(10)$

Lymphoma

$25(13)$

Myeloma

$13(6)$

Others

Status of disease at transplant

First complete response/chronic phase

Prior autologous HSCT

History of chest irradiation

$15(8)$

Donor

Related

Unrelated $^{\#}$

Stem cell source

Peripheral stem cells

Bone marrow

Cord blood

Conditioning regimen

Nonmyeloablative

$87(44)$

Total body irradiation

95 (48)

Busulfan-based

$60(30)$

Cyclophosphamide-based

$98(50)$

Antithymocyte globulin

$43(22)$

VHD prophylaxis

Cyclosporine/mycophenolate mofetil

$99(50)$

Cyclosporine/methotrexate

Other"

Cytomegalovirus status donor-recipient

Positive-positive

61 (31)

Positive-negative

$46(23)$

Negative-negative

62 (31)

Negative-positive

29 (15)

Lung CT scan findings

Abnormal $^{+}$

Air trapping score $>6$

$52(34)$

Parenchymal opacities ${ }^{\S}$

$35(23)$

Nodules

$13(9)$

Micronodules $^{f}$

$14(9)$

Bronchial thickening

Pulmonary function testing before transplant

FEV $1 \%$ pred

TLC \% pred

FVC \% pred

102 (92-112)

$100(92-110)$

100 (91-109)

SVC \% pred

$101(92-111)$

FEV $1 / F V C$

$\mathrm{FEV}_{1} / \mathrm{SVC}$

FEF25\% \% pred

$0.84(0.78-0.88)$

$90(69-117)$

FEF50\% \% pred

$99(79-116)$

FEF25-75\% \% pred

$106(85-122)$

TLCO \% pred

73 (64-81)

Normal

39 (25)

Mildly decreased

$39(25)$
$68(43)$

Moderately decreased

$47(30)$

Severely decreased 


\section{TABLE 1 Continued}

\section{Day 100 characteristics (after transplant)}

Time from HSCT to inclusion days

Karnofsky index

Current smoking

Medical history between HSCT and inclusion

Acute GVHD grade $\geqslant 2^{\# \#}$

Acute GVHD grade $\geqslant 3$

Chronic GVHD 1 १ा

Mild

Moderate/severe

LRTI

Viral URTI

Cytomegalovirus reactivation

Epstein-Barr virus reactivation

Lung CT scan findings at inclusion

Abnormal ${ }^{+}$

Air trapping score $>6$

Parenchymal opacities ${ }^{\S}$

Nodules

Bronchial abnormalities $f$

Pulmonary function testing

FEV $1 \%$ pred

TLC \% pred

FVC \% pred

FEV $1 / F V C$

FEV $1 / S V C$

FEF $25 \% \%$ pred

FEF $50 \% \%$ pred

FEF $25-75 \%$ \% pred

TLCO \% pred

LFS

Normal

01 (99-106)

$80(70-90)$

14 (7)

$80(40)$

33 (17)

46 (23)

35 (18)

$11(6)$

$18(9)$

$13(7)$

83 (42)

$40(20)$

$171(86)$

80 (47)

58 (34)

21 (12)

21 (12)

25 (15)

183 (92)

$95(85-106)$

$95(86-104)$

$93(84-106)$

$0.84(0.79-0.88)$

$0.82(0.77-0.87)$

80 (57-102)

93 (74-113)

97 (75-118)

$67(60-76)$

Mildly decreased

32 (17)

82 (45)

Moderately decreased

$57(31)$

Severely decreased

$12(7)$

Change in FEV $1 \mathrm{~mL}$

$-50(-360-112)$

Change in $\mathrm{FEV}_{1} \%$ pred

$-1.9(-10-3.5)$

$10 \%$ FEV1 decline (\% pred)

40 (28)

The data are shown as $\mathrm{n}$, median (interquartile range) or $\mathrm{n}(\%)$ unless specified. GVHD: graft versus host disease; CT: computed tomography; FEV1: forced expiratory volume in $1 \mathrm{~s}$; TLC: total lung capacity; FVC: forced vital capacity; SVC: slow vital capacity; FEF: forced expiratory flow; FEF25\%: FEF at 25\% of FVC; FEF50\%: FEF at 50\% of FVC; FEF25-75\%: FEF at 25-75\% of FVC; TLCO: transfer factor of the lung for carbon monoxide; LFS: lung function score la separate score for FEV 1 and $T$ LCO was provided: $>80 \%=1,70-80 \%=2$, $60-70 \%=3,<60 \%=4$; these scores were then summed to give the LFS and divided into four categories: normal, LFS=2; mildly decreased, LFS=3-4; moderately decreased, LFS=5-6; severely decreased, LFS=78); LRTI: lower respiratory tract infection; URTI: upper respiratory tract infection. * : 10 out of 10 and nine out of 10 allelic unrelated donors and cord blood transplants, respectively; " tacrolimus/sirolimus or methotrexate/tacrolimus; ${ }^{+}$: any lung opacities excluding air trapping; ${ }^{\S}$ : including ground-glass opacities and consolidations; ${ }^{f}$ : including centrilobular micronodules and tree-in-bud pattern; \#\#: acute GVHD usually includes erythema, maculopapular rash, nausea, vomiting, anorexia, profuse diarrhoea, ileus or cholestatic liver disease lacute GVHD was graded from 1 to 4, according to the severity, using the National Institutes of Health (NIH) consensus criteria [21]); "१?: chronic GVHD is a syndrome of variable clinical features resembling autoimmune and other immunological disorders such as scleroderma, Sjögren's syndrome, primary biliary cirrhosis, wasting syndrome, bronchiolitis obliterans, immune cytopenias and chronic immunodeficiency; manifestations of chronic GVHD may be restricted to a single organ or site or may be widespread (the global score of mild, moderate and severe reflects the degree of organ impact and functional impairment due to chronic GVHD; it has been established according to the $\mathrm{NIH}$ consensus criteria [15]).

included various diagnoses (table 2). 10 patients developed more than one LONIPC (BOS and ILD, n=2; RLD and ILD, n=1; BOS and VTED, n=1; pleural effusion and VTED, n=1; VTED and ILD, n=1; BOS and RLD, $\mathrm{n}=2$; BOS and ILD and pneumothorax, $\mathrm{n}=1$; BOS, pneumothorax and VTED, $\mathrm{n}=1$ ). 

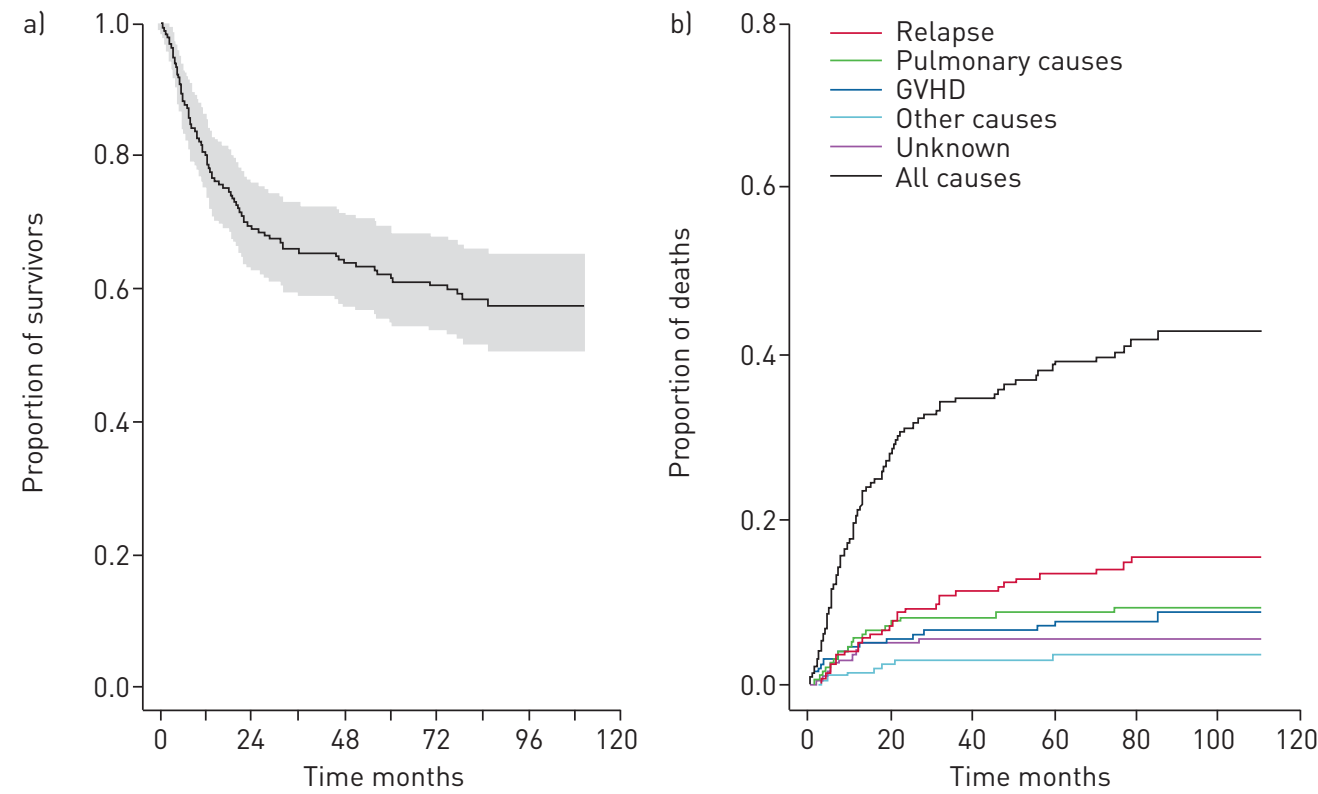

At risk $n$

$198157135127118109996134 \quad 6$

FIGURE 2 Estimated survival of allogeneic haematopoietic stem cell transplantation recipients included in the cohort (100 days after transplantation): a) overall survival $(95 \% \mathrm{Cl})$ whatever the cause of death and b) cumulative incidence of deaths according to cause. GVHD: graft versus host disease.

Most of the patients (39 out of 43 (91\%)) developed the first episode of LONIPC within 36 months (figure 3). At that time, the estimated cumulative incidence of LONIPCs was 19.8\% (95\% CI 14.2-25.3\%). All 22 episodes of BOS occurred within 36 months, with an observed median (IQR) time of $8.8(2.9-19.7)$ months after inclusion. The cumulative incidence of BOS at 36 months was $10.7 \%$ (95\% CI 6.3-15.1\%) (figure 3). Of the 22 BOS episodes, 20 (91\%) fulfilled the NIH criteria, either at the same time $(\mathrm{n}=8)$ or subsequently within a median (IQR) time of 106 (17-128) days $(\mathrm{n}=12)$.

We evaluated early predictive factors for LONIPCs diagnosed in the first 36 months after HSCT; factors selected by univariate analyses are presented in table 3 .

Multivariate models were fitted based on pre-transplant, transplant and post-transplant characteristics, separately (table 3). They exhibit the summarised predictive value of sex and history of chest irradiation before transplant, of human leukocyte antigen-related donor at transplant, and of the occurrence of a LRTI within 100 days post-transplant.

Based on multivariate Cox models that included all previously selected predictors, pre-transplant chest irradiation, LRTI before inclusion and low $\mathrm{FEF} 25-75 \%$ at inclusion were jointly associated with the occurrence of LONIPCs (table 4). Similar results were obtained for a complete case analysis.

TABLE 2 Diagnoses of late-onset noninfectious pulmonary complications (LONIPCs) observed in the sample

Bronchiolitis obliterans syndrome

Venous thromboembolic disease

Pneumomediastinum

Exudative pleural effusion

Restrictive lung defect with no interstitial lung disease or pleural disease ${ }^{\#}$

Data are presented as $\mathrm{n} .{ }^{\#}$ : including three with skin sclerosis graft versus host disease. 


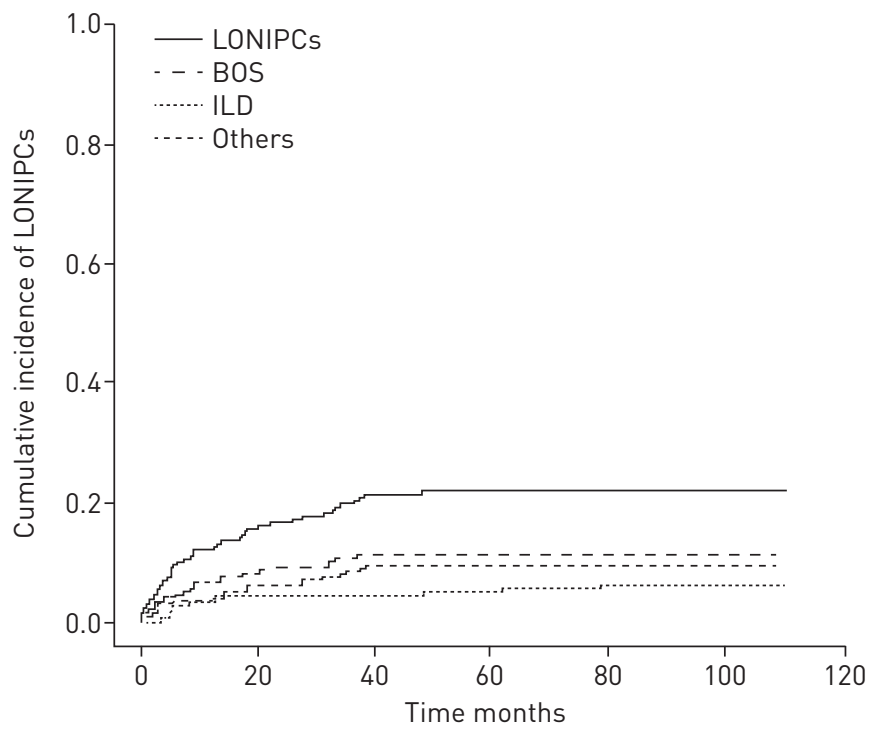

FIGURE 3 Cumulative incidence of late-onset noninfectious pulmonary complications (LONIPCs), overall and segregated into bronchiolitis obliterans syndrome (BOS), interstitial lung disease (ILD) and others.

We re-evaluated these predictors to predict BOS (table 3). Multivariate models selected the use of peripheral blood stem cells and bronchial abnormalities on the CT scan (including centrilobular micronodules, tree-in-bud pattern and bronchial thickening) at inclusion as associated with the occurrence of BOS. Based on a complete case analysis, LRTI and a 10\% FEV1 decline from baseline to day 100 were selected instead of bronchial abnormalities (table 4).

We further investigated whether the occurrence of chronic GVHD after inclusion (i.e. any time during the whole follow-up from day 100 and before the LONIPC) was predictive of LONIPCs/BOS. The cause-specific hazard of LONIPCs was increased by 2.25 (95\% CI 1.07-4.75; $\mathrm{p}=0.03$ ) after the occurrence of a chronic GVHD; that of BOS was increased by 2.90 (95\% CI 0.98-8.60; $\mathrm{p}=0.05$ ).

Among the 43 patients with LONIPCs, 18 died (nine after BOS, three after ILD, two after VTED, two after RLD, one after both BOS and RLD, and one after pneumomediastinum, BOS and ILD), with a median survival after diagnosis of 78.5 months (95\% CI 20.0-not reached). At 3 years after BOS, survival was 63.6\% (95\% CI 46.4-87.3\%) (figure 4). The occurrence of LONIPCs was associated with an increased hazard of death (HR 2.18, 95\% CI 1.14-4.15; $\mathrm{p}=0.02$ ).

At the end of follow-up (i.e. 100 months), almost $80 \%$ of the patients presented a significant PFT decrease at least once, particularly a $10 \%$ decline in FEV1 (supplementary figure S1). As depicted in supplementary figure S2, all patients who were free from LONIPCs had roughly stable FEV1 values over time, regardless of their baseline value. In contrast, the FEV1 trajectories differed among patients with BOS with two different clusters (supplementary figure S3).

\section{Discussion}

We confirmed prospectively that LONIPCs are frequent complications that largely occurred within 2 years after allogeneic HSCT with a poor outcome. Although BOS accounted for more than half of LONIPCs, these complications included heterogeneous entities, demonstrating that the risk factors for LONIPCs and BOS are distinct.

Most of the variables that we tested as risk factors have previously been associated with LONIPCs or BOS in one or more previous retrospective studies, with contradictory conclusions. As the procedures applied for allogeneic HSCT change over time, including conditioning regimens and stem cell sources, the evaluated risk factors for LONIPCs have evolved over time. Some studies have proposed that chronic GVHD is a risk factor for BOS $[6,23]$. Due to the retrospective design of these studies, we wondered whether chronic GVHD was contemporaneous to LONIPCs/BOS rather than a predictive risk factor. As expected, few patients developed chronic GVHD within 100 days following transplantation. However, prolonged follow-up of our patients led us to conclude that chronic GVHD was actually associated with the occurrence of LONIPCs. The physiopathological hypotheses of LONIPCs/BOS is that lung injury caused by various insults could be the trigger for an uncontrolled inflammatory reaction, leading to 
TABLE 3 Predictive factors for the development of bronchiolitis obliterans syndrome (BOS) and late-onset noninfectious pulmonary complications (LONIPCs) during the first 3 months after haematopoietic stem cell transplantation (HSCT): results of univariate and multivariate analyses

\begin{tabular}{|c|c|c|c|c|c|c|}
\hline & \multicolumn{3}{|c|}{ LONIPCs } & \multicolumn{3}{|c|}{ BOS } \\
\hline & $\begin{array}{c}\text { HR } \\
(95 \% \mathrm{CI})\end{array}$ & p-value & $\begin{array}{c}\text { Adjusted HR } \\
(95 \% \mathrm{Cl})\end{array}$ & $\begin{array}{c}\text { HR } \\
(95 \% \mathrm{Cl})\end{array}$ & p-value & $\begin{array}{c}\text { Adjusted HR } \\
\text { (95\% CI) }\end{array}$ \\
\hline Age at transplant years & $1.01(0.99-1.03)$ & 0.27 & & $1.01(0.99-1.04)$ & 0.32 & \\
\hline Age $>60$ years & $2.33(0.83-6.56)$ & 0.11 & & $3.27(0.97-11.06)$ & 0.06 & $2.33(0.68-8.06)$ \\
\hline Female & $0.41(0.19-0.86)$ & $0.02 *$ & $0.41(0.19-0.87)$ & $0.32(0.11-1.04)$ & $0.04^{*}$ & $0.40(0.13-1.23)$ \\
\hline History of autologous HSCT & $1.47(0.72-3.03)$ & 0.29 & & $1.28(0.47-3.47)$ & 0.63 & \\
\hline History of chest irradiation & $3.63(1.59-8.29)$ & $0.00 *$ & $3.63(1.58-8.31)$ & $2.51(0.74-8.52)$ & 0.14 & \\
\hline \multicolumn{7}{|l|}{ Pulmonary function testing } \\
\hline FEV1 $\%$ pred $/ 10 \%$ & $0.91(0.72-1.15)$ & 0.42 & & $0.92(0.67-1.25)$ & 0.58 & \\
\hline TLC \% pred/10\% & $0.86(0.66-1.13)$ & 0.29 & & $0.99(0.69-1.41)$ & 0.94 & \\
\hline TLCo $\%$ pred $/ 10 \%$ & $0.99(0.70-1.40)$ & 0.95 & & $0.93(0.59-1.46)$ & 0.74 & \\
\hline Air trapping score $>6$ & $0.89(0.38-2.07)$ & 0.79 & & $1.61(0.60-4.29)$ & 0.34 & \\
\hline \multicolumn{7}{|l|}{ Transplant data (day 0) } \\
\hline Disease status (first complete response) & $1.23(0.65-2.31)$ & 0.53 & & $1.09(0.47-2.52)$ & 0.84 & \\
\hline Cytomegalovirus status & $0.71(0.38-1.34)$ & 0.29 & & $0.71(0.31-1.64)$ & 0.42 & \\
\hline \multicolumn{7}{|l|}{ Source of cells } \\
\hline HLA-related & $0.53(0.28-1.00)$ & $0.05^{*}$ & $0.44(0.23-0.85)$ & $0.81(0.35-1.86)$ & 0.62 & \\
\hline PBSCs & $2.36(1.08-5.13)$ & $0.03^{*}$ & $1.58(0.64-3.94)$ & $6.11(1.43-26.1)$ & $0.01 *$ & $6.11(1.43-26.1)$ \\
\hline Cord blood & $1.24(0.38-4.04)$ & 0.72 & & $0.72(0.10-5.32)$ & 0.74 & \\
\hline \multicolumn{7}{|l|}{ Conditioning } \\
\hline Nonmyeloablative & $1.72(0.91-3.23)$ & 0.09 & $1.61(0.68-3.83)$ & $1.32(0.57-3.05)$ & 0.51 & \\
\hline Cyclophosphamide-based & $1.97(1.02-3.79)$ & $0.04^{*}$ & $1.24(0.46-3.35)$ & $1.91(0.80-4.56)$ & 0.14 & \\
\hline Antithymocyte globulin & $0.45(0.18-1.15)$ & 0.09 & $0.38(0.14-1.01)$ & 0 & & \\
\hline Acute GVHD grade $\geqslant 3$ & $1.73(0.82-3.65)$ & 0.15 & & $0.81(0.24-2.74)$ & 0.74 & \\
\hline Chronic GVHD+ & $0.72(0.32-1.63)$ & 0.43 & & $0.33(0.08-1.41)$ & 0.14 & \\
\hline Moderate/severe chronic GVHD & $0.63(0.09-4.56)$ & 0.64 & & 0 & & \\
\hline LRTI within 100 days post-transplant & $3.87(1.77-8.45)$ & $0.00 *$ & $2.94(1.04-7.90)$ & $3.81(1.40-10.4)$ & $0.01 *$ & $2.34(0.72-7.58)$ \\
\hline \multicolumn{7}{|l|}{ Pulmonary function testing } \\
\hline FEV $1 \%$ pred $/ 10 \%$ & $0.74(0.62-0.88)$ & $0.00 *$ & $0.95(0.71-1.27)$ & $0.75(0.61-0.94)$ & $0.01 *$ & $0.94(0.67-1.32)$ \\
\hline TLC $\%$ pred/10\% & $0.83(0.67-1.04)$ & 0.09 & & $0.95(0.71-1.26)$ & 0.71 & \\
\hline TLCo $\%$ pred $/ 10 \%$ & $1.02(0.80-1.31)$ & 0.85 & & $1.10(0.81-1.49)$ & 0.54 & \\
\hline $\mathrm{FEF}_{25-75 \%} \%$ pred $/ 10 \%$ & $0.81(0.72-0.91)$ & $0.00 *$ & $0.82(0.61-1.09)$ & $0.83(0.71-0.97)$ & $0.02 *$ & $0.85(0.61-1.18)$ \\
\hline FEF50\% \% pred/10\% & $0.83(0.74-0.94)$ & $0.00^{*}$ & $1.07(0.79-1.46)$ & $0.83(0.71-0.98)$ & $0.02 *$ & $1.04(0.74-1.47)$ \\
\hline Decreased LFS & $0.77(0.35-1.67)$ & 0.51 & & $0.52(0.20-1.34)$ & 0.18 & \\
\hline Severely decreased LFS & $2.21(1.01-4.81)$ & $0.05^{*}$ & $1.00(0.30-3.28)$ & $0.73(0.17-3.14)$ & 0.68 & \\
\hline $5 \%$ FEV1 decline from baseline ${ }^{\S}$ & $3.20(1.46-6.99)$ & $0.00^{*}$ & $1.19(0.32-4.42)$ & $2.25(0.84-6.04)$ & 0.11 & $0.42(0.05-3.14)$ \\
\hline $10 \% \mathrm{FEV}_{1}$ decline from baseline $\mathrm{s}^{\S}$ & $4.21(1.97-9.01)$ & $0.00 *$ & $1.41(0.36-5.49)$ & $3.89(1.45-10.45)$ & $0.01 *$ & $2.64(0.37-18.75)$ \\
\hline
\end{tabular}


TABLE 3 Continued

\begin{tabular}{|c|c|c|c|c|c|c|}
\hline & \multicolumn{3}{|c|}{ LONIPCs } & \multicolumn{3}{|c|}{ BOS } \\
\hline & $\begin{array}{c}\text { HR } \\
(95 \% \mathrm{Cl})\end{array}$ & p-value & $\begin{array}{c}\text { Adjusted HR } \\
(95 \% \mathrm{CI})\end{array}$ & $\begin{array}{c}\text { HR } \\
(95 \% \mathrm{Cl})\end{array}$ & p-value & $\begin{array}{c}\text { Adjusted HR } \\
\text { (95\% CI) }\end{array}$ \\
\hline \multicolumn{7}{|l|}{ Lung CT scan } \\
\hline Normal & $0.53(0.28-1.01)$ & $0.05^{*}$ & $1.04(0.46-2.37)$ & $0.49(0.21-1.14)$ & 0.10 & \\
\hline Air trapping score $>6$ & $1.28(0.66-2.46)$ & 0.47 & & $1.53(0.66-3.54)$ & 0.32 & \\
\hline Bronchial abnormalities $f$ & $2.58(1.13-5.94)$ & $0.02 *$ & $1.22(0.46-3.22)$ & $6.03(2.40-15.10)$ & $0.00^{*}$ & $3.00(1.06-8.48)$ \\
\hline \multicolumn{7}{|c|}{$\begin{array}{l}\text { FEV1: forced expiratory volume in } 1 \mathrm{~s} \text {; TLC: total lung capacity; TLCO: transfer factor of the lung for carbon monoxide; FEF: forced expiratory } \\
\text { flow; FEF25\%: FEF at } 25 \% \text { of FVC; FEF50\%: FEF at } 50 \% \text { of FVC; FEF } 25-75 \% \text { : FEF at } 25-75 \% \text { of FVC; LFS: lung function score; CT: computed } \\
\text { tomography; HLA: human leukocyte antigen; PBSC: peripheral blood stem cell; GVHD: graft versus host disease. }{ }^{\#} \text { after multiple imputation } \\
\uparrow_{\text {: }} \text { see table } 1 \text { for details of acute GVHD; }{ }^{+} \text {: see table } 1 \text { for details of chronic GVHD; }{ }^{\S} \text { : as measured by absolute differences in } \% \text { predicted } \\
f_{\text {: including centrilobular micronodules, tree-in-bud pattern and bronchial thickening. }}^{*} \text { : significant at } p \leqslant 0.05 \text {. }\end{array}$} \\
\hline
\end{tabular}

aberrant tissue repair [24]. We found that a history of chest irradiation and the occurrence of early pneumonia after HSCT were strongly predictive of LONIPCs/BOS, reinforcing this hypothesis.

The diagnosis of post-transplant BOS using noninvasive methods is challenging [24]. In the early 2000s, lung CT emerged as a promising tool to diagnose BOS $[25,26]$. Some authors have even suggested that the presence of air trapping on expiratory scans could precede PFT impairment $[27,28]$. These data led to the inclusion of air trapping as a diagnostic criterion for post-allogeneic HSCT BOS in the NIH consensus $[13,15]$. We were surprised to find that nearly a quarter of patients had significant air trapping before transplantation. This phenomenon could be attributed to the observation that allogeneic HSCT recipients are older and have a history of respiratory events explaining the air trapping, including respiratory infection during a prior course of chemoradiotherapy. Furthermore, although the proportion of patients with significant air trapping increased at day 100 after transplant, air trapping was not predictive of BOS. Conversely to air trapping, bronchial abnormalities at day 100, including centrilobular micronodules, tree-in-bud pattern and bronchial thickening, were predictive of BOS. This finding is of particular interest because these CT scan abnormalities could reflect the early phase of inflammatory bronchiolitis obliterans when treatment could be effective.

TABLE 4 Predictive factors for the development bronchiolitis obliterans syndrome (BOS) and late-onset noninfectious pulmonary complications (LONIPCs) during the first 3 months after haematopoietic stem cell transplantation (HSCT): results of multivariate analyses

\begin{tabular}{|c|c|c|}
\hline & $\operatorname{HR}(95 \% \mathrm{CI})$ & p-value \\
\hline \multicolumn{3}{|l|}{ LONIPCs } \\
\hline \multicolumn{3}{|c|}{ Multivariate analysis after multiple imputation (198 patients, 39 LONIPCs) } \\
\hline Chest irradiation prior to HSCT & $3.60(1.57-8.28)$ & 0.0025 \\
\hline History of LRTI within 100 days after HSCT & $3.12(1.38-7.07)$ & 0.0065 \\
\hline Low FEF25-75\% at day 100 & $0.84(0.75-0.94)$ & 0.0034 \\
\hline \multicolumn{3}{|c|}{ Multivariate analysis on complete cases (182 patients, 36 LONIPCs) } \\
\hline Chest irradiation prior to HSCT & $3.12(1.29-7.57)$ & 0.0118 \\
\hline History of LRTI within 100 days after HSCT & $2.57(1.04-6.33)$ & 0.0407 \\
\hline Low FEF25-75\% at day 100 & $1.21(0.73-0.94)$ & 0.0025 \\
\hline \multicolumn{3}{|c|}{ BOS } \\
\hline \multicolumn{3}{|c|}{ Multivariate analysis after multiple imputation (198 patients, 22 BOS) } \\
\hline PBSCs & $6.07(1.40-26.2)$ & 0.0158 \\
\hline History of LRTI within 100 days after HSCT & $3.28(1.20-8.88)$ & 0.0198 \\
\hline Bronchial abnormalities on CT scan at day 100 & $2.96(1.02-8.57)$ & 0.0449 \\
\hline \multicolumn{3}{|c|}{ Multivariate analysis on complete cases (144 patients, 16 BOS) } \\
\hline PBSCs & $7.69(1.00-58.9)$ & 0.0495 \\
\hline $10 \% \mathrm{FEV}_{1}$ decline between baseline and day 100 & $3.07(1.14-8.29)$ & 0.0271 \\
\hline
\end{tabular}




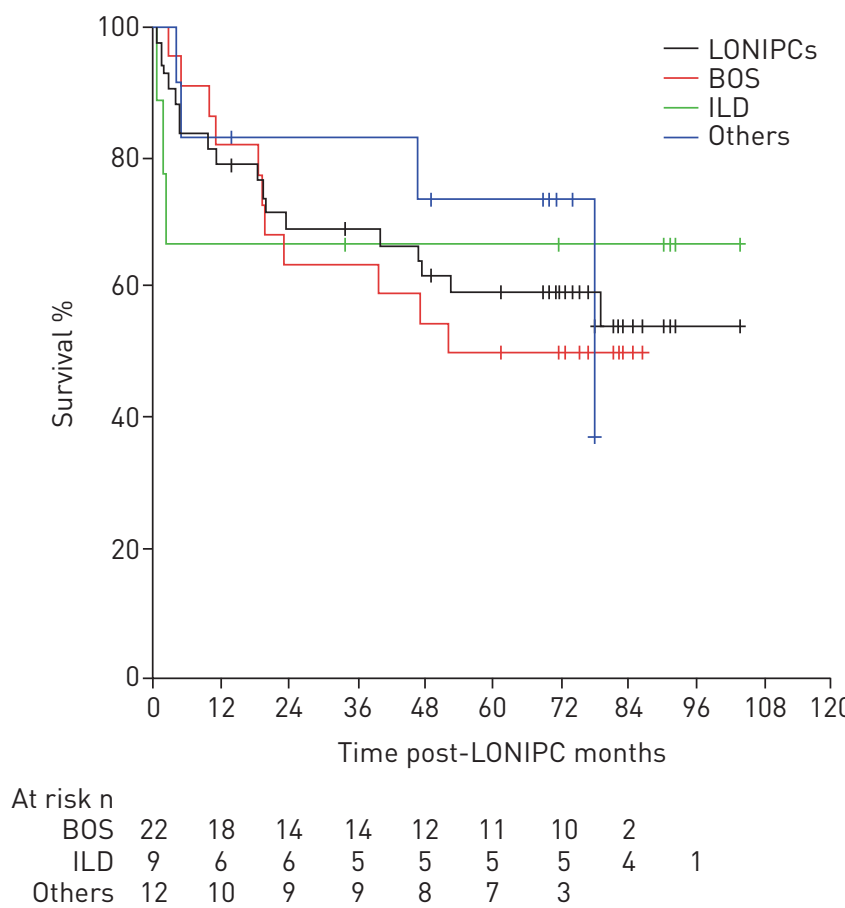

FIGURE 4 Overall survival after late-onset noninfectious pulmonary complications (LONIPCs), overall and segregated into bronchiolitis obliterans syndrome (BOS), interstitial lung disease (ILD) and others.

To date, PFT is the cornerstone of BOS diagnosis [15]. However, the current criteria do not allow for consideration of all BOS $[4,29,30]$. Recently, Thompson et al. [3] showed that the median change in FEV1 from pre-transplant to day 100 in patients who subsequently developed LONIPCs was significantly greater than that in unaffected patients. We found that a decrease in airflow at day 100, both in proximal and small airways (i.e. FEV1 and FEF), was predictive of LONIPCs/BOS. In particular, a $10 \%$ decline in FEV 1 from pre-transplant to day 100 was predictive of BOS in the multivariate analysis. These findings strongly encourage monitoring spirometry early after an allogeneic HSCT and suggest consideration of the trajectory of FEV1 rather than the absolute value at a given time. This finding is consistent with the findings of CHENG et al. [31], who observed a rapid FEV1 decline during the 6 months prior to BOS diagnosis.

Our prospective cohort provides a unique opportunity to assess allogeneic HSCT recipient lung function over a long follow-up period. Thus, we have shown that lung function fluctuated and that a very significant proportion of patients experienced a significant decline in airflow at least once during their follow-up. However, FEV1 or FEF mostly returned to baseline values in the follow-up PFT. The many events that occur after transplant can explain this issue, especially respiratory infections and a transient deterioration in the general condition that jeopardises the quality of spirometric performance.

Finally, we provide important data regarding BOS. We used a definition that differs from that of the NIH consensus, which allowed an earlier diagnosis of BOS. Of note, we used this definition in previous studies $[4,20]$. In addition, we identified two phenotypes of BOS according to the trajectory of FEV 1 with different outcomes. This result raises the question of a different pathophysiological mechanism and whether different approaches for management should be investigated.

Our study has several limitations. First, due to the small number of patients who experienced LONIPCs other than BOS, we could not reliably evaluate the risk factors for each specific LONIPC. Second, our study was a single-centre study; however, given that all PFT was performed at the same place using the same plethysmograph, this reinforces the value of our PFT variations findings by reducing inaccuracy and avoiding potential bias incurred by PFT performed using different machines. Nevertheless, the single-centre recruitment limits the external validity required to support widespread changes in practice; results should thus apply at the bedside only after comparing the context of the study with the context of clinical experience. Third, some data were missing, especially some PFT and CT scans during forced expiration, which were difficult to obtain in patients with poor general health. However, missing data were imputed with no main modifications of the results. Indeed, the various predictive factors for BOS that were identified either after multiple imputation (MICE) or multivariate analysis of the complete cases were 
actually correlated (data not shown). Finally, we did not collect data to allow calculating scores that were shown to be independently predictive of increased mortality after allogeneic HSCT, such as the haematopoietic cell transplantation-specific comorbidity index or the disease risk index [32, 33]. Thus, this issue precluded their assessment in our predictive models for LONIPCs. This could be explored in further studies.

In conclusion, our data provide clues to identify patients with a high risk of developing LONIPCs. These patients should be targeted for close monitoring, especially PFT, and thus offered earlier treatment of LONIPCs or prophylactic treatment to improve outcomes.

Acknowledgements: The authors thank Stéphane Cassonnet and Emmanuelle Bugnet (Hôpital Saint-Louis, Paris, France) for the clinical study coordination and technical support, Nathalie Chemla and Stéphane Beziaud (Clinique du Sport, Paris, France) for performing the lung CT scans, Françoise Grondin (Hôpital Saint-Louis) for performing the pulmonary function tests, and Elisabeth Savariau (Hôpital Saint-Louis) for excellent technical assistance. We also thank all HSCT recipients who participated in the study and EGMOS, an association of bone marrow transplant recipients. In memory of Laurène, an allogeneic HSCT recipient, for her energy, her help and her strong desire to advance research on LONIPCs.

Conflict of interest: A. Bergeron has received personal fees for lectures from Gilead and Pfizer, and personal fees for lectures and board participation from Merck, outside the submitted work. R. Peffault de Latour has received personal fees and grants from Alexion, Novartis and Pfizer, and research grants from Amgen, outside the submitted work. K. Chagnon has received personal fees from AstraZeneca, outside the submitted work.

Support statement: This study was supported by an institutional grant from the French Ministry of Health (CRC 04118), which had no role in the study design, data collection, data analysis, data interpretation or writing of the report. The corresponding author had full access to the data in the study and the final responsibility for the decision to submit the study findings for publication.

\section{References}

1 Patriarca F, Skert C, Bonifazi F, et al. Effect on survival of the development of late-onset non-infectious pulmonary complications after stem cell transplantation. Haematologica 2006; 91: 1268-1272.

2 Ueda K, Watadani T, Maeda E, et al. Outcome and treatment of late-onset noninfectious pulmonary complications after allogeneic haematopoietic SCT. Bone Marrow Transplant 2010; 45: 1719-1727.

3 Thompson PA, Lim A, Panek-Hudson Y, et al. Screening with spirometry is a useful predictor of later development of noninfectious pulmonary syndromes in patients undergoing allogeneic stem cell transplantation. Biol Blood Marrow Transplant 2014; 20: 781-786.

4 Bergeron A, Godet C, Chevret S, et al. Bronchiolitis obliterans syndrome after allogeneic hematopoietic SCT: phenotypes and prognosis. Bone Marrow Transplant 2013; 48: 819-824.

5 Schlemmer F, Chevret S, Lorillon G, et al. Late-onset noninfectious interstitial lung disease after allogeneic hematopoietic stem cell transplantation. Respir Med 2014; 108: 1525-1533.

6 Au BK, Au MA, Chien JW. Bronchiolitis obliterans syndrome epidemiology after allogeneic hematopoietic cell transplantation. Biol Blood Marrow Transplant 2011; 17: 1072-1078.

7 Bergeron A. Late-onset noninfectious pulmonary complications after allogeneic hematopoietic stem cell transplantation. Clin Chest Med 2017; 38: 249-262.

8 Palmas A, Tefferi A, Myers JL, et al. Late-onset noninfectious pulmonary complications after allogeneic bone marrow transplantation. Br J Haematol 1998; 100: 680-687.

9 Patriarca F, Skert C, Sperotto A, et al. Incidence, outcome, and risk factors of late-onset noninfectious pulmonary complications after unrelated donor stem cell transplantation. Bone Marrow Transplant 2004; 33: 751-758.

10 Sakaida E, Nakaseko C, Harima A, et al. Late-onset noninfectious pulmonary complications after allogeneic stem cell transplantation are significantly associated with chronic graft-versus-host disease and with the graft-versus-leukemia effect. Blood 2003; 102: 4236-4242.

11 Solh $\mathrm{M}$, Arat $\mathrm{M}$, Cao $\mathrm{Q}$, et al. Late-onset noninfectious pulmonary complications in adult allogeneic hematopoietic cell transplant recipients. Transplantation 2011; 91: 798-803.

12 Dharmagunawardena R, Pearce RM, Thomas G, et al. A retrospective multi-centre study of the effects of allogeneic haematopoietic SCT on pulmonary function. Bone Marrow Transplant 2014; 49: 1442-1443.

13 Filipovich $\mathrm{AH}$, Weisdorf $\mathrm{D}$, Pavletic S, et al. National Institutes of Health consensus development project on criteria for clinical trials in chronic graft-versus-host disease: I. Diagnosis and staging working group report. Biol Blood Marrow Transplant 2005; 11: 945-956.

14 Chien JW, Duncan S, Williams KM, et al. Bronchiolitis obliterans syndrome after allogeneic hematopoietic stem cell transplantation - an increasingly recognized manifestation of chronic graft-versus-host disease. Biol Blood Marrow Transplant 2010; 16: S106-S114.

15 Jagasia MH, Greinix HT, Arora M, et al. National Institutes of Health consensus development project on criteria for clinical trials in chronic graft-versus-host disease: I. The 2014 diagnosis and staging working group report. Biol Blood Marrow Transplant 2015; 21: 389-401.

16 European Respiratory Society. Standardized lung function testing. Official statement of the European Respiratory Society. Eur Respir J Suppl 1993; 16: 1-100.

17 Parimon T, Madtes DK, Au DH, et al. Pretransplant lung function, respiratory failure, and mortality after stem cell transplantation. Am J Respir Crit Care Med 2005; 172: 384-390.

18 Lee ES, Gotway MB, Reddy GP, et al. Early bronchiolitis obliterans following lung transplantation: accuracy of expiratory thin-section CT for diagnosis. Radiology 2000; 216: 472-477. 
19 Arakawa H, Webb WR. Air trapping on expiratory high-resolution CT scans in the absence of inspiratory scan abnormalities: correlation with pulmonary function tests and differential diagnosis. AJR Am J Roentgenol 1998; 170: 1349-1353.

20 Bergeron A, Chevret S, Chagnon K, et al. Budesonide/formoterol for bronchiolitis obliterans after hematopoietic stem cell transplantation. Am J Respir Crit Care Med 2015; 191: 1242-1249.

21 Przepiorka D, Weisdorf D, Martin P, et al. 1994 Consensus Conference on Acute GVHD Grading. Bone Marrow Transplant 1995; 15: 825-828.

22 Genolini C, Falissard B. KmL: a package to cluster longitudinal data. Comput Methods Programs Biomed 2011; 104: e112-e121.

23 Ditschkowski M, Elmaagacli AH, Koldehoff M, et al. Bronchiolitis obliterans after allogeneic hematopoietic SCT: further insight - new perspectives? Bone Marrow Transplant 2013; 48: 1224-1229.

24 Barker AF, Bergeron A, Rom WN, et al. Obliterative bronchiolitis. N Engl J Med 2014; 370: $1820-1828$.

25 Siegel MJ, Bhalla S, Gutierrez FR, et al. Post-lung transplantation bronchiolitis obliterans syndrome: usefulness of expiratory thin-section CT for diagnosis. Radiology 2001; 220: 455-462.

26 Gunn ML, Godwin JD, Kanne JP, et al. High-resolution CT findings of bronchiolitis obliterans syndrome after hematopoietic stem cell transplantation. J Thorac Imaging 2008; 23: 244-250.

27 Bankier AA, Van Muylem A, Knoop C, et al. Bronchiolitis obliterans syndrome in heart-lung transplant recipients: diagnosis with expiratory CT. Radiology 2001; 218: 533-539.

28 de Jong PA, Dodd JD, Coxson HO, et al. Bronchiolitis obliterans following lung transplantation: early detection using computed tomographic scanning. Thorax 2006; 61: 799-804.

29 Uhlving HH, Andersen CB, Christensen IJ, et al. Biopsy-verified bronchiolitis obliterans and other noninfectious lung pathologies after allogeneic hematopoietic stem cell transplantation. Biol Blood Marrow Transplant 2015; 21: 531-538.

30 Holbro A, Lehmann T, Girsberger S, et al. Lung histology predicts outcome of bronchiolitis obliterans syndrome after hematopoietic stem cell transplantation. Biol Blood Marrow Transplant 2013; 19: 973-980.

31 Cheng GS, Storer B, Chien JW, et al. Lung function trajectory in bronchiolitis obliterans syndrome after allogeneic hematopoietic cell transplantation. Ann Am Thorac Soc 2016; 13: 1932-1939.

32 Sorror ML, Maris MB, Storb R, et al. Hematopoietic cell transplantation (HCT)-specific comorbidity index: a new tool for risk assessment before allogeneic HCT. Blood 2005; 106: 2912-2919.

33 Armand P, Gibson CJ, Cutler C, et al. A disease risk index for patients undergoing allogeneic stem cell transplantation. Blood 2012; 120: 905-913. 